\title{
iMTracker: um programa para monitoramento remoto das ações de usuários de sistemas Web
}

\author{
Reginaldo do Prado ${ }^{1}$, Leônidas O. Brandão ${ }^{2}$, Anarosa A. F. Brandão ${ }^{3}$ \\ ${ }^{1}$ Instituto Federal de Educação, Ciência e Tecnologia de São Paulo - (IFSP), \\ ${ }^{2}$ Instituto de Matemática e Estatística - Universidade de São Paulo (IME-USP), \\ ${ }^{3}$ Escola Politécnica - Universidade de São Paulo (EP-USP) \\ rprado@ifsp.edu.br, leo@ime.usp.br, anarosa.brandao@poli.usp.br
}

\begin{abstract}
Understanding the way people interact with on-line systems during learning is an important issue to improve quality of both learning and systems. Monitoring the user's actions while interacting with web-based systems is a common activity carried on to promote systems' improvement. Several tools are available to support them, but they usually are not free software. In this paper we present iMTracker, a free tool for monitoring the users' interaction with web-based systems tailored to support web-based systems. In addition, we discuss some aspects related to the monitoring of web-based systems considering educational purposes.
\end{abstract}

Resumo. Entender como as pessoas interagem com os sistemas computacionais para aprendizagem on-line é importante para melhorar sua qualidade. O monitoramento de ações dos usuários de páginas Web é comum na indústria e fornece dados que podem ajudar seus desenvolvedores a melhorar a usabilidade de aplicações baseadas na Web, mas geralmente isso é feito com o uso de software comercial. Neste artigo apresentamos iMTracker, um software livre para monitoramento de interações de usuários com sistemas on-line dedicados à educação. Além disso, discutimos alguns aspectos relacionados ao monitoramento de sistemas on-line para apoiar a educação.

\section{Introdução}

A Web oferece uma grande oportunidade para a avaliação rápida de ideias com o uso de experimentos on-line controlados [Kohavi, Hene and Somerfield, 2007]. Também chamados de experimentos aleatórios ou testes $\mathrm{A} / \mathrm{B}$, estes procedimentos têm influenciado vários campos do conhecimento como medicina, agricultura, manufatura e propaganda [Crook et al, 2009]. Neste tipo de teste cria-se uma versão experimental do software, chamada de tratamento (treatment) e confronta-se os resultados de seu uso com aqueles coletados sobre o uso de uma versão pré-existente, chamada de controle (control) para avaliar a ideia [Kohavi, Hene and Somerfield, 2007]. A principal 
V Congresso Brasileiro de Informática na Educação (CBIE 2016)

Anais do XXVII Simpósio Brasileiro de Informática na Educação (SBIE 2016)

vantagem deste tipo de abordagem é a possibilidade de o usuário final de um sistema praticamente guiar a sua evolução, resultando em um produto mais bem adaptado às necessidades de seu consumidor [Kohavi, Hene and Somerfield, 2007]. Acreditamos que o desenvolvimento de software educacional pode se beneficiar desta abordagem para aumentar a qualidade das interações dos professores e dos estudantes com o software educacional, culminando em uma intervenção positiva sobre os processos de ensino e de aprendizagem como um todo.

Para entender como ocorre atualmente o uso deste tipo de teste no desenvolvimento de software educacional realizamos uma revisão da literatura e encontramos 32 trabalhos sobre desenvolvimento de software que mencionam o uso de algum experimento on-line para coleta de dados sobre o comportamento do usuário ao utilizar o sistema. Esta pesquisa foi realizada na base de dados do Institute of Electrical and Electronic Engineers [IEEExplore, 2016] e revisada em 14/05/2016. Dos sistemas analisados nos artigos da consulta, nenhum estava diretamente ligado ao ensino ou à aprendizagem.

Ainda, buscando entender porque os experimentos on-line são pouco utilizados na área acadêmica, fizemos buscas na Web para identificar quantas e quais são as ferramentas que estão disponíveis para este tipo de trabalho. Notamos que existe uma grande diversidade de opções, das quais destacamos apenas o Clicktale [Clicktale, 2016] e o Inspectlet [Inspectlet, 2016] que permitem o uso das técnicas mais comuns de monitoramento de interação do usuário com o sistema Web, que são: contagem de cliques, mapeamento dos movimentos do cursor, mapeamento da rolagem $\mathrm{e}$ redimensionamento da página e geração de mapas de calor (heatmaps) - imagens que usam cores quentes e frias para indicar, respectivamente, as regiões mais visitadas e as menos visitadas pelo cursor. Destacamos o Clicktale e o Inspectlet porque ambos implementam as técnicas básicas de monitoramento mencionadas acima.

Devido ao grande número de empresas que usam esse tipo de ferramenta com finalidade comercial, praticamente todas as opções disponíveis são proprietárias. Não encontramos ferramentas livres (nem gratuitas) para a coleta on-line de dados sobre o uso de sistemas Web. Neste artigo, apresentamos o sistema iMTracker como alternativa livre para o monitoramento das interações do usuário de software educacional executado no ambiente Web.

$\mathrm{Na}$ próxima seção fazemos uma breve revisão da literatura e procuramos estabelecer o estado da arte em experimentos on-line. Na seção 3 descrevemos o sistema iMTracker, suas características e seu estado de desenvolvimento. Na seção 4 apresentamos uma discussão e fechamos o artigo na seção 5, com as conclusões e indicação de trabalhos futuros. 
V Congresso Brasileiro de Informática na Educação (CBIE 2016)

Anais do XXVII Simpósio Brasileiro de Informática na Educação (SBIE 2016)

\section{Monitoramento como instrumento para entender o usuário}

$\mathrm{O}$ uso de experimentos on-line tem seus primeiros registros no início do século XXI, com o trabalho de Le e Waseda (2000). Estes pesquisadores criaram o Curious Browser, um navegador Web que registrou as atividades de 75 alunos navegando por mais de 2500 páginas. Eles descobriram que o tempo de movimento do cursor sobre uma página pode ser usado para indicar a relevância da página para o usuário.

Chen, Anderson e Sohn (2001), estudaram o relacionamento entre a direção do olhar e as posições do cursor. Eles mostraram que a distância entre o olhar e a posição do cursor era notavelmente pequena. Esta técnica tornou-se interessante por permitir que pesquisadores determinassem em quais regiões da página o usuário está mais interessado sem ter que levar o usuário a um laboratório e gravar seus movimentos em vídeo abrindo caminho para que tais experimentos sejam realizados em larga escala. Huang, White and Dumais (2011) examinaram o comportamento do cursor em páginas de mecanismos de busca incluindo não apenas cliques, mas também os movimentos e sobreposições do mouse (mouse hover) em diferentes regiões da página. Eles reportaram que a posição do cursor está diretamente ligada à direção do olhar do usuário, principalmente em páginas de resultados de busca.

Kohavi, Hene e Somerfield (2007) analisaram quais seriam os requisitos mais importantes para a execução de experimentos on-line controlados. Eles descreveram as arquiteturas de experimentação mais comuns e analisaram suas vantagens e desvantagens. Guo e Agichtein (2010) usando um navegador modificado encontraram diferenças entre as distâncias percorridas pelo mouse correlacionando com o tipo de busca realizada pelo usuário.

\section{3. iMTracker}

O sistema iMTracker foi proposto para possibilitar o rastreamento do uso do mouse por parte de um usuário, para avaliar um sistema produzido autoria de cursos Web via Moodle, projeto iPlan [Prado, Brandão e Brandão, 2014].

O iMTracker está sendo implementado em JavaScript (2016), podendo ser adicionado a qualquer ambiente $W e b$, particularmente permitindo o monitoramento de sistemas Web que apoiem a educação. Ele registra a movimentação do mouse, permitindo a reprodução dos movimentos do cursor e mapeamento dos cliques do mouse sobre regiões específicas de páginas web.

O iMTracker segue uma arquitetura cliente/servidor, que é descrita na figura 1. No lado do cliente, o iMTracker provê funcionalidades de monitoramento bem como de relatórios de monitoramento. Durante o monitoramento, ele recebe as informações de interação do usuário com o sistema, extraindo coordenadas e armazenando pares de informação (ponto, hora) até que a capacidade de armazenamento local atinja um determinado limite. Toda informação armazenada é enviada ao servidor, que a insere 
V Congresso Brasileiro de Informática na Educação (CBIE 2016)

Anais do XXVII Simpósio Brasileiro de Informática na Educação (SBIE 2016)

num banco de dados. O relatório de monitoramento é gerado no lado servidor e enviado para o cliente, que gera um mapa de pontos ou uma animação do movimento do cursor.

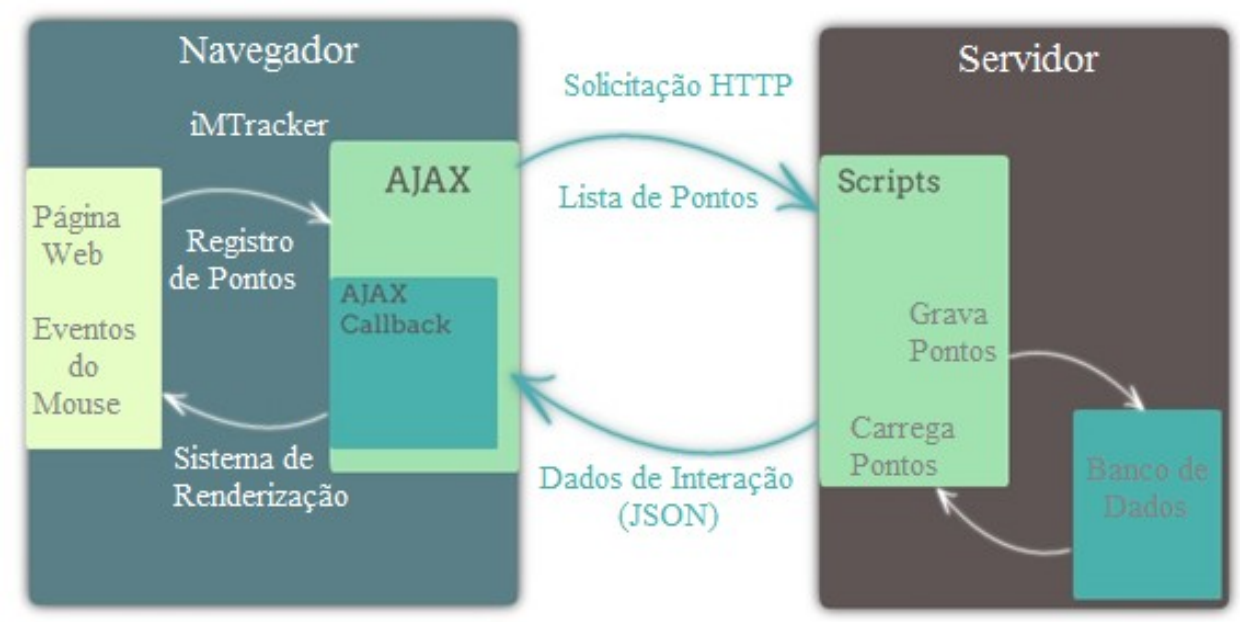

Figura 1. Arquitetura do iMTracker

O rastreamento do mouse permite ao desenvolvedor visualizar conjuntos de segmentos de reta, sobrepostos à página web, determinados pelos movimentos do usuário, indicando as posições por onde o cursor foi deslocado. Os mesmos pontos que determinam os segmentos de reta do rastreamento do mouse também são usados para a exibição de uma animação do cursor na tela que permite visualizar os movimentos realizados pelo usuário, passo a passo, ao longo do tempo, em um processo similar à exibição de um filme dos movimentos do usuário. Naturalmente, os movimentos do cursor são entremeados por cliques e estes também são registrados pelo iMTracker, permitindo que o desenvolvedor visualize pontos coloridos marcados sobre as regiões da página web onde o usuário clicou.

O iMTracker pode registrar de forma concomitante e independente as interações dos usuários sobre as regiões de uma página, de modo que duas ou mais regiões da mesma página possam ser analisadas no mesmo experimento. Além disso, os dados sobre a interação de cada usuário são gravados em um banco de dados, o que possibilita ao pesquisador que usa o iMTracker a realização de experimentos em larga escala pois, teoricamente, não existe limite para a quantidade de interações registradas pelo sistema.

Adicionalmente deve-se destacar que o iMTracker está sendo desenvolvido na forma de software livre, por exemplo, disponível a partir da área do Laboratório de Informática na Educação (LInE) no GitHub (https://github.com/LInE-IMEUSP/iMTracker).

\section{Discussão}

O iMTracker é uma alternativa livre para monitoramento de sistemas Web, em particular para sistemas de apoio ao ensino e à aprendizagem via web. Ele pode beneficiar várias audiências, como os desenvolvedores de sistemas Web e professores que adotam 
V Congresso Brasileiro de Informática na Educação (CBIE 2016)

Anais do XXVII Simpósio Brasileiro de Informática na Educação (SBIE 2016)

sistemas Web para apoiar seus cursos, sejam eles à distância ou mistos.

No contexto da Informática na Educação, entendemos que a realização de experimentos on-line em protótipos de sistemas Web concebidos para apoiar o ensino e a aprendizagem podem conduzir melhorias no sistema final. Além disso, experimentos deste tipo podem guiar a evolução de sistemas Web existentes, melhorando interfaces e, eventualmente, facilitando o ensino e a aprendizagem on-line. Na figura 2 apresentamos uma imagem resultante do iMTracker para rastrear o uso de um professor com o iPlan [Prado, Brandão, 2014].

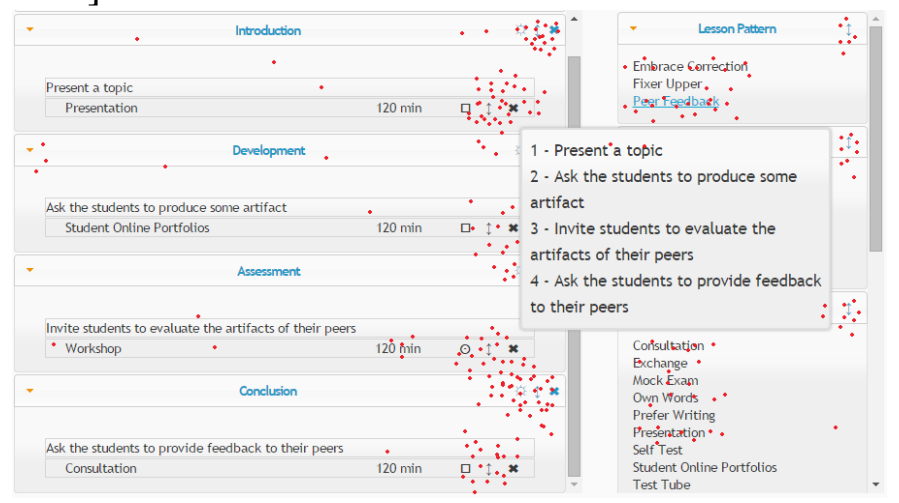

Figura 2. Imagem de uso do iMTracker para analisar o uso do iPlan.

Ainda, professores cujas disciplinas possam se beneficiar de acompanhamento das ações do aluno durante sua interação com alguma atividade proposta via sistema também são usuários em potencial do iMTracker. Como exemplo, podemos citar um professor que ministra geometria com apoio de algum sistema Web. Ainda que estes sistemas armazenem um $\log$ com as ações dos alunos, a visualização da animação da interação do aluno com o sistema é um recurso adicional para entender o raciocínio que este aluno seguiu enquanto resolvia determinado problema.

\section{Conclusão e trabalhos futuros}

Neste trabalho apresentamos o sistema iMTracker, que permite monitorar remotamente as interações de visitantes de uma página ou um sistema Web. Seu desenvolvimento foi motivado pela escassez de ferramentas livres para que pesquisadores pudessem realizar experimentos on-line relacionados com alguns itens de usabilidade.

Atualmente seu desenvolvimento se encontra em estágio testes e revisão e já pode ser usado para registrar cliques, monitorar movimentos do mouse e reproduzir as ações dos usuários como uma animação. Brevemente deveremos desenvolver novas funcionalidades, como rastreamento da ação "arrastar", criação de mapas de calor e recursos associados (como geração da imagem associada ao mapa). Uma versão inicial do iMTracker está disponível na área do LInE-IME-USP no GitHub (https://github.com/LInE-IME-USP/iMTracker) e serão contribuições serão bem vindas! 
V Congresso Brasileiro de Informática na Educação (CBIE 2016)

Anais do XXVII Simpósio Brasileiro de Informática na Educação (SBIE 2016)

\section{References}

ClickTale (2016) http://www.clicktale.com/ Acesso em 12/06/2016

Chen, M.C., Anderson, J.R. and Sohn, M.H. (2001). "What can a mouse cursor tell us more?: correlation of eye/mouse movements on web browsing". Extended Abstracts on Human Factors in Computing Systems - CHI '01, 281-282.

Crook, T., Frasca, B., Kohavi R. and Longbotham, R. (2009) "Seven Pitfalls to Avoid when Running Controlled Experiments on the Web". 15st ACM SIGKDD Conference on Knowledge Discovery and Data Mining - Paris, France.

Deng, A., Xu, Y. and Kohavi, R. (2013). "Improving the Sensitivity of On-line Controlled Experiments by Utilizing Pre-Experiment Data". Proceedings of the sixth ACM international conference on Web search and data mining Pages - WSDM '13.

Goecks, J. and Shavlik, J. (2000) "Learning users' interests by unobtrusively observing their normal behavior". Proceedings of the 5th international conference on Intelligent user interfaces - IUI'00, 129-132.

Guo, Q. and Agichtein, E. (2010). "Exploring mouse movements for inferring query intent". Proceedings of the 31st annual international ACM SIGIR conference on Research and development in information retrieval - SIGIR'10, 707-708.

Hijikata, Y. (2004) "Implicit user profiling for on demand relevance feedback". Proceedings of the 9th international conference on Intelligent user interfaces.

Hong, L. and Ying, C. (2009) "How Webpage Background Affect On-line Shopping Behavior". 3st IEEE International Conference on Management and Service Science, 2009. MASS'09 - Beijing, China.

Huang, J., White, R. W., and Dumais, S. (2011). "No Clicks, No Problem: Using Cursor Movements to Understand and Improve Search". Proceedings of the SIGCHI Conference on Human Factors in Computing Systems - SIGCHI'11.

Inspectlet (2016) https://www.inspectlet.com. Acesso em 12/6/2016.

IEEExplore (2016) http://ieeexplore.ieee.org/Xplore/home.jsp. Acesso em 12/6/2016.

Javascript (2016) http://www.w3.org/standards/webdesign/script. Acesso em 12/6/2016.

Kohavi, R., Henne, R. M., Sommerfiled, D. (2007) "Practical Guide to Controlled Experiments on the Web: Listen to Your Customers not to the HiPPO". 13st ACM SIGKDD Conference on Knowledge Discovery and Data Mining - San Jose, CA.

Le, P. and Waseda, M. (2000) "A Curious Browser: Implicit Ratings". Fulfillment of the requirements for the Degree of Bachelor Science at Worcester Polytechnic Institute.

Prado, R., Brandão, L.O. and Brandão, A.A.F. (2014) "iPlan: a model of lesson plans to help teaching process in Web learning environment". 2014 IEEE Frontiers in Education Conference (FIE) Proceedings. 